\title{
ARTICLE OPEN \\ Intraoperative delivery of the Notch ligand Jagged-1 regenerates appendicular and craniofacial bone defects
}

Daniel W. Youngstrom ${ }^{1}$, Rafael Senos ${ }^{1}$, Robert L. Zondervan ${ }^{1,2}$, Jack D. Brodeur ${ }^{2}$, Austin R. Lints ${ }^{2}$, Devin R. Young ${ }^{2}$, Troy L. Mitchell ${ }^{1}$, Megan E. Moore ${ }^{2}$, Marc H. Myers ${ }^{3}$, Wei-Ju Tseng ${ }^{3}$, Kathleen M. Loomes ${ }^{4}$ and Kurt D. Hankenson (iD)

Each year, $33 \%$ of US citizens suffer from a musculoskeletal condition that requires medical intervention, with direct medical costs approaching \$1 trillion USD per year. Despite the ubiquity of skeletal dysfunction, there are currently limited safe and efficacious bone growth factors in clinical use. Notch is a cell-cell communication pathway that regulates self-renewal and differentiation within the mesenchymal/osteoblast lineage. The principal Notch ligand in bone, Jagged-1, is a potent osteoinductive protein that positively regulates post-traumatic bone healing in animals. This report describes the temporal regulation of Notch during intramembranous bone formation using marrow ablation as a model system and demonstrates decreased bone formation following disruption of Jagged-1 in mesenchymal progenitor cells. Notch gain-of-function using recombinant Jagged-1 protein on collagen scaffolds promotes healing of craniofacial (calvarial) and appendicular (femoral) surgical defects in both mice and rats. Localized delivery of Jagged-1 promotes bone apposition and defect healing, while avoiding the diffuse bone hypertrophy characteristic of the clinically problematic bone morphogenetic proteins. It is concluded that Jagged-1 is a bone-anabolic agent with therapeutic potential for regenerating traumatic or congenital bone defects.

npj Regenerative Medicine (2017)2:32; doi:10.1038/s41536-017-0037-9

\section{INTRODUCTION}

Fractures, congenital defects, and malignancies of the cranial, axial, and appendicular skeleton present a common challenge; however, current therapies aimed at promoting bone regeneration have significant shortcomings. By the age of $50,50 \%$ of women and $20 \%$ of men will have suffered a fracture. ${ }^{1}$ Up to $12 \%$ of tibia shaft fractures experience nonunion, ${ }^{2}$ and approximately $25 \%$ of bone allografts to repair the resection of malignant bone tumors fail. ${ }^{3}$ Indeed, the direct medical costs of osteoporosisrelated fractures alone in the United States exceeds $\$ 20$ billion annually. ${ }^{4}$ The total societal burden of these injuries, which includes healthcare utilization, reduced quality/quantity of life and opiate abuse, will continue to rise with ageing and the prevalence of comorbidities. ${ }^{5}$ The bone morphogenetic proteins (BMPs) were once championed as versatile skeletal growth factors and the answer to the bone healing problem, but the widespread off-label clinical use of BMPs contributed to an increasingly poor safety record and a pronounced drop in their utilization. Thus, there remains a major unmet public health need for alternative and/or complimentary bone-anabolic factors that can promote healing of high-risk fractures or volumetric bone defects.

Notch is an evolutionarily conserved cell-cell signaling pathway that, in mammals, has five ligands (Jagged-1, Jagged-2, Delta-like1, Delta-like-3, Delta-like-4) and four receptors (Notch1-4). Upon ligand binding, Notch receptors activate by proteolytic cleavage of their intracellular domains (NICD), which translocate to the nucleus where they function as transcription factors for a variety of genes, including those involved in musculoskeletal differentiation ${ }^{7}$ (Supplementary Fig. S1). Mutations in the Jagged-1
(Jag1) or Notch2 genes cause Alagille syndrome in humans, an autosomal dominant disorder with characteristic skeletal manifestations, including osteopenia and high prevalence of fractures. $^{8}$ Dominant-positive Notch2-activating mutations cause Hajdu-Cheney syndrome, which also has a strong skeletal phenotype. ${ }^{9}$ Interest in the role of the Notch pathway during bone development and healing has grown dramatically over the past decade, with the majority of experimental data gathered in mice. However, mechanistic interpretation of transgenic mouse models has been controversial in the field, and is confounded by variables related to experimental protocol, cell lineage state, signal strength, and microenvironment.

Genetic deletion of Jag1 in mice is embryonic lethal, ${ }^{10}$ and canonical RBPjk-mediated Notch signaling regulates the activity of skeletal stem cells during development. ${ }^{11}$ Notch inhibition in limb bud mesenchyme (Prx1-Cre;Psen $1^{\mathrm{f} / \mathrm{f}} \mathrm{Psen} 2^{-/-}$or Prx1-Cre;Notch1 ${ }^{-/ \mathrm{f}}$ Notch $2^{\mathrm{f} / \mathrm{f}}$ ) causes stunting of the skeleton with an expanded cartilaginous growth plate, a decrease in marrow stem cell number and a reduction in osteoblast marker gene expression in vitro. ${ }^{12}$ Deletion of the dominant ligand, Jag1 $\left(\operatorname{Prx} 1-C r e ; J a g 1^{\mathrm{f} / \mathrm{f}}\right)$, dysregulates the osteochondral progenitor cell pool and produces compartment-specific phenotypes including femoral trabecular osteopenia in adulthood. ${ }^{13}$ Notch2 deletion in early osteoblasts (Runx2-Cre;Notch2 $2^{\mathrm{f} / \mathrm{f}}$ ) increases bone volume (BV) in selected regions. ${ }^{14}$ In committed osteoblasts, Notch1 overactivation (Col1a1-Cre;N1ICD ${ }^{\mathrm{Tg}}$ ) causes hypertrophic intramedullary osteosclerosis, and Notch inactivation (Col1a1-Cre;:Psen $1^{\mathrm{f} / \mathrm{f}}$ Psen $2^{-/}$) results in vertebral and appendicular osteopenia. ${ }^{15}$

\footnotetext{
${ }^{1}$ Orthopaedic Research Laboratories, Department of Orthopaedic Surgery, University of Michigan Medical School, Ann Arbor, MI, USA; ${ }^{2}$ Department of Physiology, Michigan State University College of Osteopathic Medicine, East Lansing, MI, USA; ${ }^{3}$ Department of Orthopaedic Surgery, University of Pennsylvania Perelman School of Medicine, Philadelphia, PA, USA and ${ }^{4}$ Division of Gastroenterology, Hepatology and Nutrition, The Children's Hospital of Philadelphia, Philadelphia, PA, USA Correspondence: Kurt D. Hankenson (kdhank@umich.edu)
}

Received: 17 August 2017 Revised: 7 November 2017 Accepted: 21 November 2017

Published online: 15 December 2017 
In both the endochondral long bone fracture environment and during calvarial intramembranous bone healing, analyses of whole-callus mRNA and immunohistochemistry suggest that canonical Jag1/Notch2 signaling drives differentiation of postinjury mesenchyme from days five through $20 .{ }^{16}$ Notch blockade (Mx1-Cre;dnMAML ${ }^{\mathrm{f} / \text { ) }}$ ) alters fracture callus remodeling by day 20 but does not impair early callus formation. ${ }^{17}$ However, Notchrelated mRNA is downregulated at days two and six post-fracture in aSMA + progenitor cells, and NICD overexpression impairs the osteogenic differentiation of this population in vitro. ${ }^{18}$ Pan-Notchinactivated mesenchyme (Prx1-Cre;RBPjk ${ }^{\mathrm{f} / \mathrm{f}}$ ) deposits a larger, more cartilaginous callus that never properly remodels to bone, resulting in sustained nonunion through 42 days. ${ }^{19}$

Taken together, there is likely a spatiotemporal switch that occurs midway through the continuum of osteoblast differentiation. Early activation of Notch drives progenitor cell proliferation and inhibits entrance into the early osteochondral phase, whereas mid-to-late activation of Notch drives osteoblast differentiation and promotes the anabolic activity of committed osteoblasts. Activating Notch in primed, post-injury mesenchyme represents a potential targeted strategy to increase bone formation.

Mammalian Notch ligands require physical association with a cell/object in order to effectively signal: soluble Notch ligands are competitive antagonists to the pathway. ${ }^{20}$ Jag1 can be immobilized onto a scaffold or carrier to induce osteoblast differentiation, and we have previously demonstrated feasibility of this approach in vitro using human primary cell lines. ${ }^{21,22}$ The goal of this study was to further define the regulatory role of Notch signaling in osteoblast differentiation and intramembranous bone healing in vivo, and to explore the therapeutic utility of Jag1 to promote bone anabolism. Our hypothesis was that Jag1 would promote osteoblastogenesis of mesenchymal progenitor cells and drive bone regeneration in experimentally induced bone defects in rodents. Herein, we demonstrate that Jag1 is a safe and effective local therapeutic for bone repair. Disruption of Jag1 during bone formation reduces bone healing, and delivery of Jag1 using clinically applicable collagen sponges significantly enhances bone healing without excess ectopic bone.

\section{RESULTS}

Jag1 expression occurs concurrently with increased osteoblast differentiation genes during intramembranous bone formation Our previous work has shown that Jag1 initiated canonical Notch signaling is osteoanabolic in vitro using human mesenchymal stem cells, ${ }^{21,22}$ and that Jag1 disruption in vivo in osteoblast lineage cells decreases femoral trabecular bone. ${ }^{13}$ To more fully probe the role of Jag1 and Notch signaling in adult intramembranous bone formation, we used marrow ablation as a model that results in pronounced compartmentalized intramembranous bone formation. Marrow ablation reduced bone volume fraction (BV/TV) by $58.9 \%$ to $0.052 \pm 0.033(p=0.057)$, followed by a spike in bone formation peaking at day 7 , at which time there was $224.3 \%$ more BV/TV than basal bone $(0.286 \pm 0.049, p<0.001)$, with $37.4 \%$ higher bone mineral density (BMD) $(324.7 \pm 38.0 \mathrm{mg} /$ $\mathrm{CC}, p=0.039$ ) (Fig. 1a). Mean BV and bone mineral content (BMC) also increased. aSMA-positive mesenchymal cells and their progeny were the architects of this response, representing the dominant cell population in the regenerating region and colocalizing with fluorescent bone labels (Fig. 1b). The destruction and regeneration of intramedullary bone was followed histologically (Supplementary Fig. S2), with a blood clot forming within hours, followed by activation and proliferation of mesenchymal cells, presumably from endosteal and/or periosteal niches. The intramedullary compartment was filled with differentiating mesenchyme from days 2-4 post ablation, with the anabolic osteoblastic response peaking at day 7 , followed by a catabolic phase.

In uninjured bone, JAG1 and NOTCH2 represent 95.2 and $70.3 \%$ of total marrow Notch ligand and receptor transcript, respectively (Supplementary Fig. S3). These remain the dominant Notch species throughout healing. Following marrow ablation, JAG1 was significantly upregulated at days 1-6 (Fig. 1), peaking with a $2.87 \pm 1.65$-fold increase in expression over basal bone at day three $(p<0.001)$. Male mice expressed a $12.0 \pm 5.2 \%$ greater foldchange in JAG1 versus females at this timepoint $(p=0.013)$. Peak fold-change values for JAG2, DLL1, DLL3, and DLL4 occurred at days 6 (NS), $3(p=0.005), 4 \quad(p=0.004)$ and $2(p<0.001)$, respectively. Males exhibited statistically lower DLL1 expression $(p=0.002)$, while there were no sex differences in peak levels of JAG2, DLL3 or DLL4. NOTCH1 and 2 were downregulated and NOTCH3 and 4 were upregulated during the later stages of bone formation, with the greatest fold-change observed in the least abundant receptor, NOTCH4. Fold-change of NOTCH1 and 2 expression was $7.30-23.1 \%$ lower in males than females from days 7-9 $(p=0.002-0.047)$.

Expression of RUNX2, an essential osteoblast transcription factor, peaked at day 3 with $1.79 \pm 0.69$-fold expression ( $p=$ $0.002)$ and $10.4 \pm 5.7 \%$ more expression in males than females ( $p$ $=0.018)$. ACTA2 ( $\mathrm{aSMA}$ ) expression, indicative of mesenchymal progenitor activation, peaked at day 2 with $5.08 \pm 3.79$-fold expression $(p=0.002)$ and $21.6 \pm 4.2 \%$ more expression in males than females $(p=0.007)$. There was sustained upregulation of the inflammatory marker CCL7 $(p=0.002)$ and downregulation of the chondrogenic gene COL2A1 $(p<0.001)$ starting at day 1 . IBSP expression peaked at days $3-4(p<0.001$ and $p=0.032$, respectively), with greater expression in females than males $(p<0.001)$. LFNG, MFNG and RFNG were not differentially regulated for more than one day (not shown).

Jag1, but not Jag2, is required for peak intramembranous bone formation

To interrogate the role of Jag1 during intramembranous bone regeneration, we disrupted Jag1 in the early mesenchyme using inducible Cre-mediated recombination of the floxed Jag1 gene (loxP sites flanking exons 4-5), with Cre driven from the aSMA promoter (Fig. 1b). Jag1 knockout in aSMA-expressing cells at the time of bone injury (aSMA-Cre ${ }^{\text {ERT2}} ;$ Jag $^{f / f}$ ) resulted in a $25.7 \%$ reduction in $\mathrm{BV} / \mathrm{TV}$ to $0.269 \pm 0.056(p=0.021)$ and a $12.4 \%$ reduction in BMD to $327.9 \pm 29.9 \mathrm{mg} / \mathrm{cc}(p=0.032)$ at time of peak bone mass in day 7 females, relative to tamoxifen-treated, Crepositive controls with wild-type Jag1 alleles (Fig. 2). Jag2 knockout (loxP sites flanking exons 1-2) using the same inducible Cre did not result in changes in BMD $(p=0.276)$ or BV/TV $(p=0.403)$ (Supplementary Fig. S4). Histologically, Jag1 knockout mice developed a dense network of intramedullary trabeculae by day 7, but the intramedullary compartment contained cavities of marrow and regions of incomplete mineralization relative to their Cre-negative counterparts (Fig. 2).

Recombinant Jag1 improves healing of craniofacial and long bone defects in mice

Our previous in vitro research has shown that recombinant Jag1 is an osteoanabolic factor: ${ }^{21,22}$ thus, we examined whether Jag1 could promote bone regeneration in vivo. Delivery of Jag1 protein to calvarial defects in mice increased bone regeneration. Representative $\mu C T$ isosurfaces demonstrated new bone induced by treatment with Jag1 ( $\mu$ C-Jag1) or BMP2 (GF-BMP2) versus microcarrier/Gelfoam ( $\mu \mathrm{C}-\mathrm{Vh}$ ) and Gelfoam (GF-Vh) controls (Fig. 3). GF-Vh grafting resulted in $0.934 \pm 0.513 \mathrm{~mm}^{3}$ of new bone at day 42 , compared with $1.222 \pm 0.463 \mathrm{~mm}^{3}$ for $\mu \mathrm{C}-\mathrm{Vh}, 1.722 \pm 0.586 \mathrm{~mm}^{3}$ for $\mu \mathrm{C}$-Jag 1 and $2.876 \pm 1.603 \mathrm{~mm}^{3}$ for GF-BMP2. Relative to the corresponding $\mu \mathrm{C}-\mathrm{Vh}$ control, within the entire $3.3 \times 3.3 \times 1.7 \mathrm{~mm}$ 

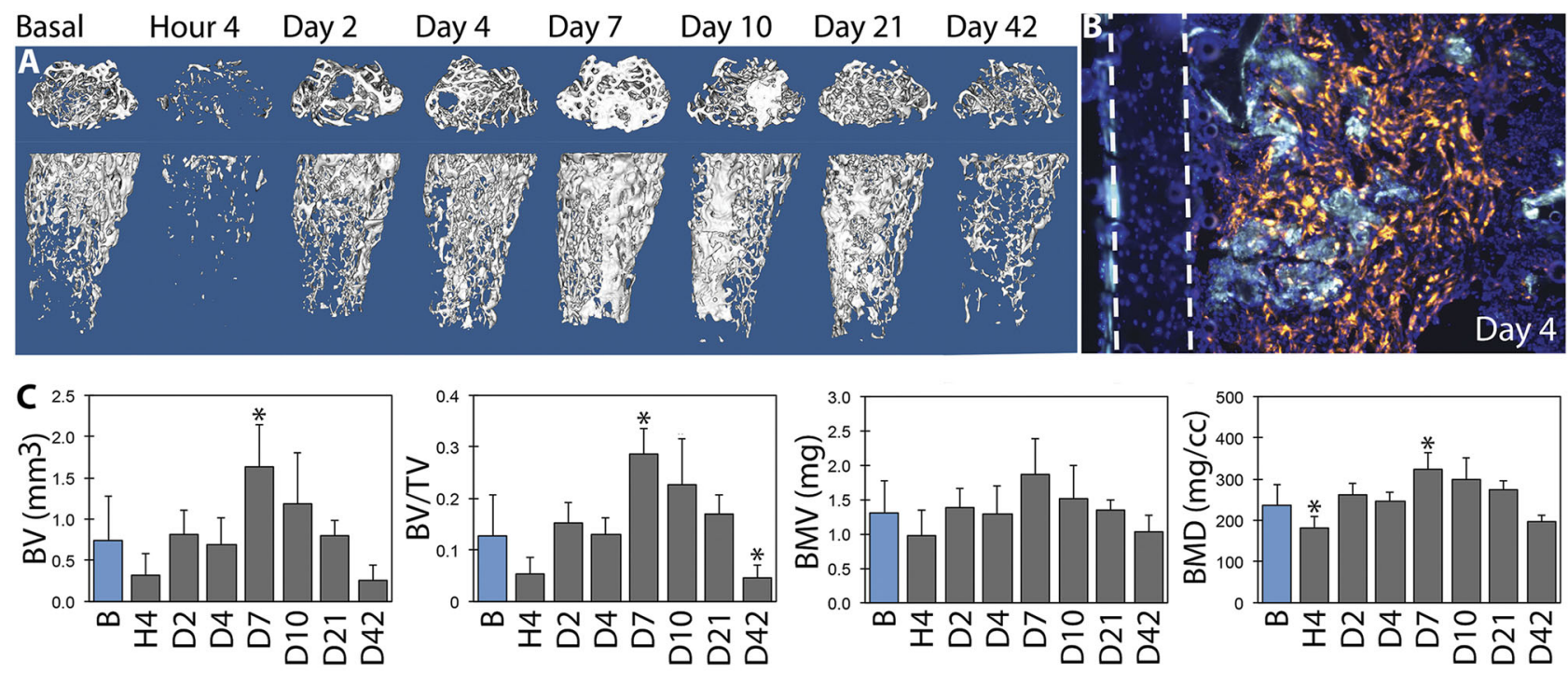

D
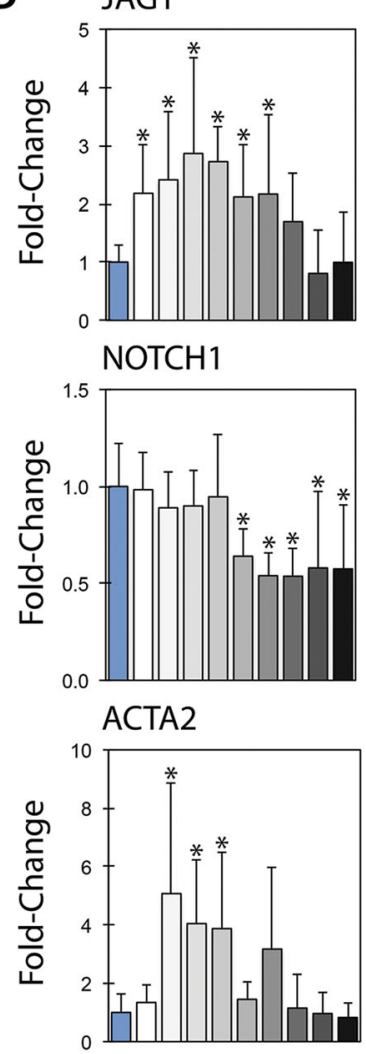

JAG2

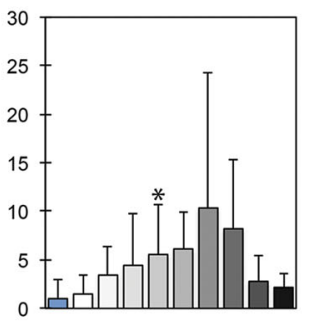

$\mathrm{NOTCH} 2$

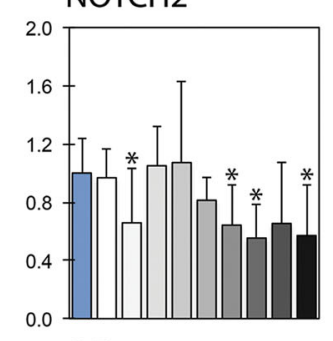

CCL7

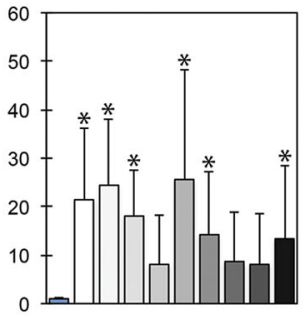

DLL1

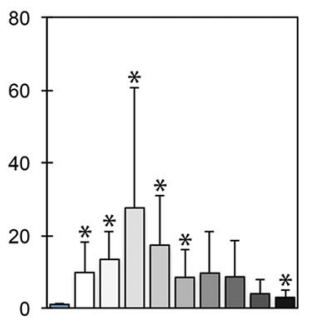

$\mathrm{NOTCH} 3$

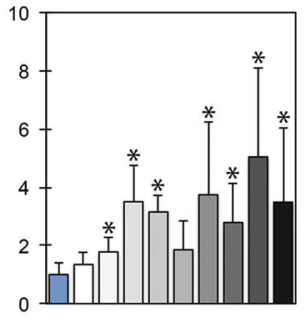

COL2A1

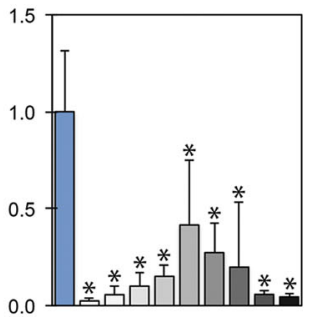

DLL3

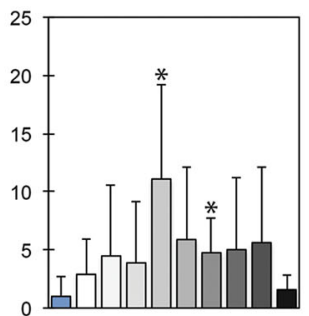

$\mathrm{NOTCH} 4$

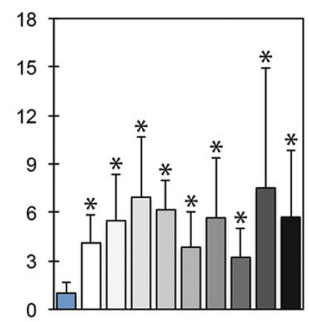

IBSP

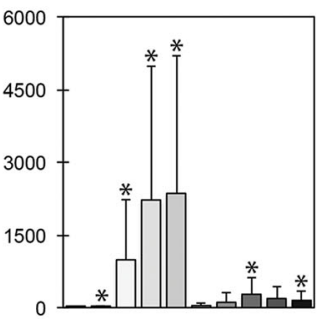

DLL4

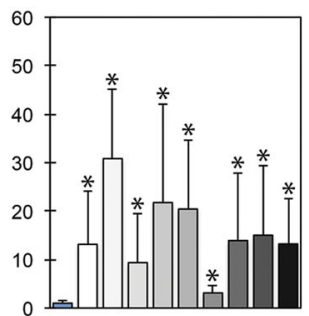

RUNX2

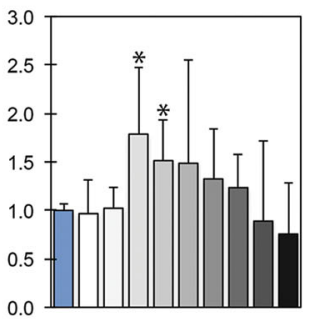

口Basal

口Day 1

口Day 2

口Day 3

-Day 4

口Day 5

口Day 6

口Day 7

口Day 8

-Day 9

Fig. $1 \mu C T$ and qPCR profile post-marrow ablation. Mechanical disruption of the marrow environment results in deposition of intramedullary bone within seven days. This was quantified by $\mu \mathrm{CT}(n=6)$, with representative sagittal and coronal 3D reconstructions shown (a, c). Osteoblasts derived from $\alpha$ SMA-positive mesenchymal progenitor cells produce new (double-labeled) bone: the cortical wall is demarcated with dashed lines (b). Expression of Notch ligands, receptors, and select marker genes are presented over time by qPCR with respect to basal bone, relative to ACTB (d). Notch ligands are upregulated during osteoblast differentiation. Statistical significance is symbolized with * for $p<$ $0.05(n=6-12)$

cylindrical region of interest (ROI) surrounding the defect, the $\mu \mathrm{C}$ Jag1 group developed $37.5 \%$ more BV to $0.828 \pm 0.222 \mathrm{~mm}^{3}(p=$ $0.008), 39.8 \%$ more tissue mineral content to $0.586 \pm 0.163 \mathrm{mg}(p$ $=0.007)$ and $37.4 \%$ greater BV/TV to $0.0576 \pm 0.0154(p=0.008)$. There were near-significant increases in BMC $(p=0.077)$ and BMD $(p=0.079)$. Tissue mineral density was not changed $(p=0.677)$. Importantly, the new bone formed in the $\mu \mathrm{C}$-Jag1 group remained in the plane of the defect (Fig. 4), while in contrast, BMP2 treatment caused production of new bone that extended well above the injury margin. Marrow (with adipose) and encapsulated intact Gelfoam were observed within the regenerated bone only in the GF-BMP2 group. aSMA-positive mesenchymal cells were labeled at the time of treatment by administering tamoxifen to aSMA-Cre ${ }^{\text {ERT2 }}$; tdTomato mice. There was a greater quantity of aSMA-positive mesenchymal cells and their progeny in the $\mu \mathrm{C}$ Jag1 group versus the $\mu \mathrm{C}-\mathrm{Vh}$ group, showing significantly 

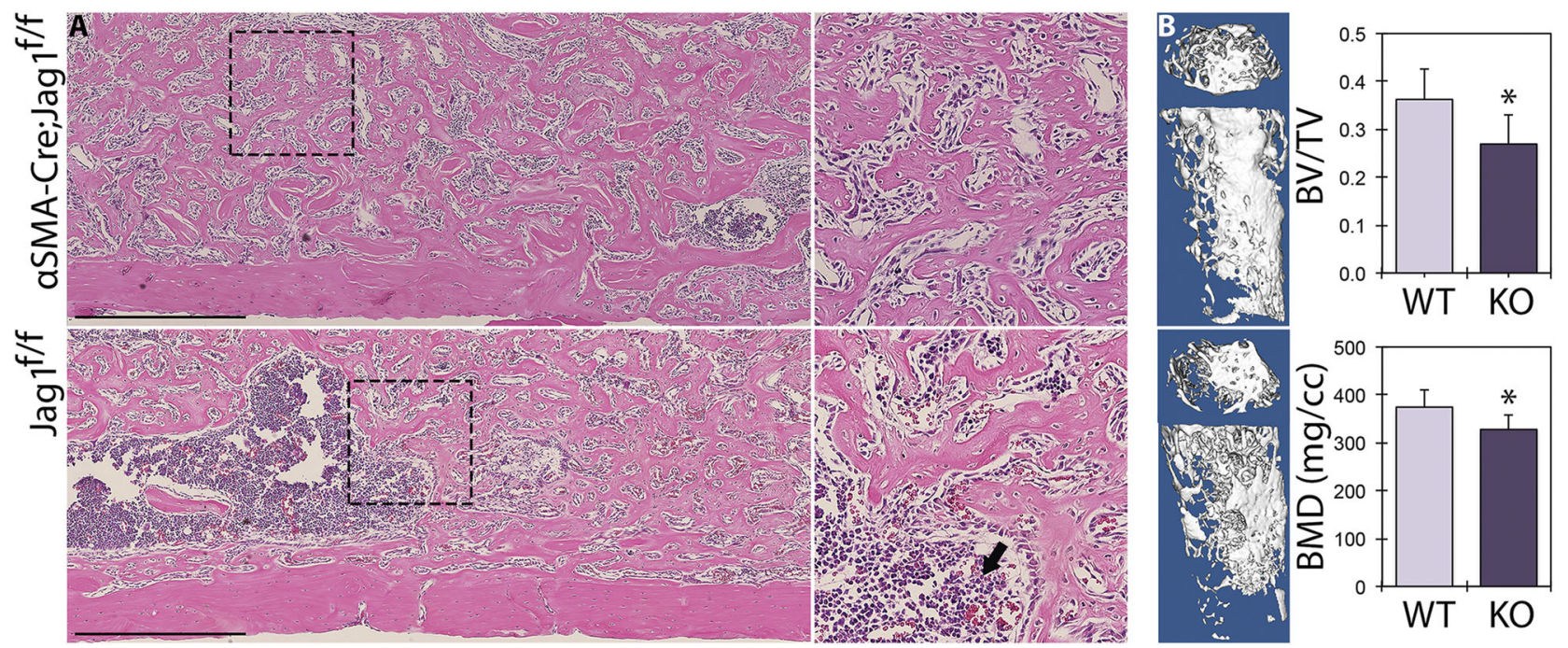

Fig. 2 Jag1-KO reduces post-ablation bone formation. Cre-positive and Cre-negative female littermates in the Jag $1^{\mathrm{f} / \mathrm{f}}$ line $(n=3)$ underwent marrow ablation procedures and received single tamoxifen injections at time of surgery. H\&E histology (a) is shown alongside $\mu C T$ reconstructions and quantitation (b) for day 7 post ablation. Jag1-KO animals underwent incomplete osteoblastogenesis and contain regions of marrow interspersed within the newly formed trabecular bone (arrow). Scale bars are equal to $500 \mu \mathrm{mm}$. Statistical significance is symbolized with * for $p<0.05$

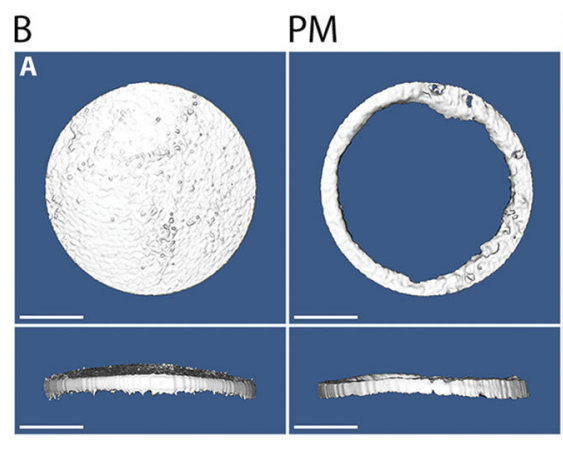

GF Vh

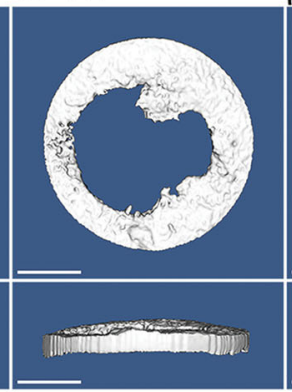

$\mu \mathrm{C}$ Jag-1

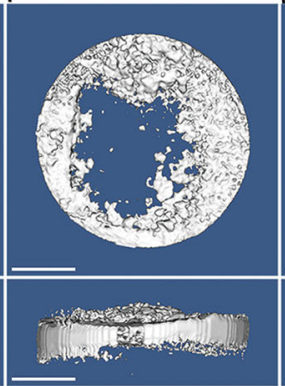

$\mu \mathrm{CJag}-1$

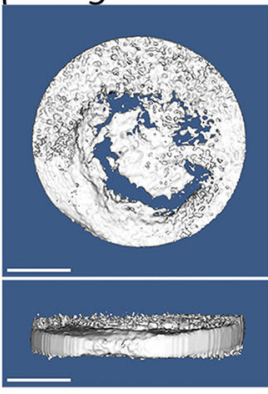

GF BMP-2

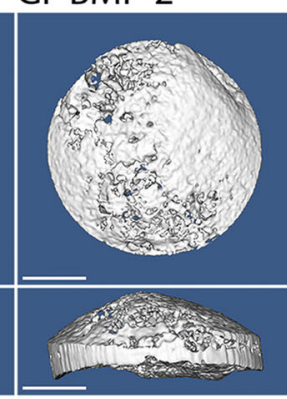

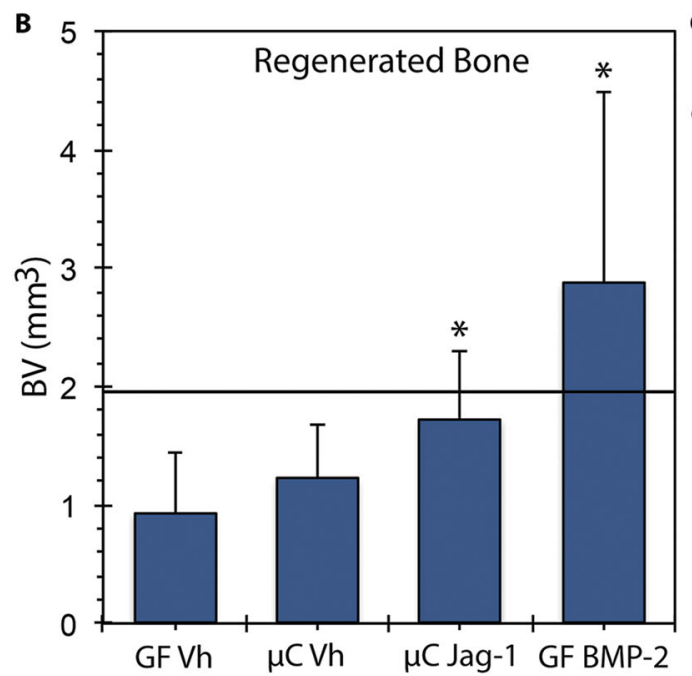
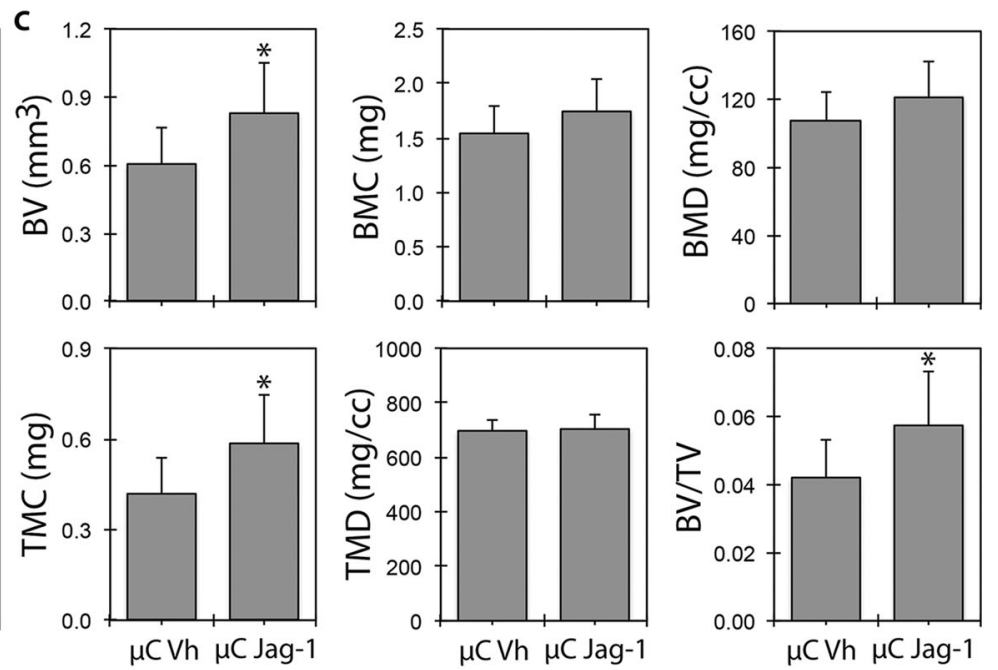

Fig. 3 Jag1 improves healing of calvarial defects in mice. 3D reconstructions (a) and total regenerated bone (b) (PM-subtracted) were assessed using $\mu \mathrm{CT} 42$ days post-defect, reported at a threshold of 666. Total ROI quantitation between $\mu \mathrm{C}-\mathrm{Vh}$ and $\mu \mathrm{C}$-Jag 1 groups (c) was conducted at a threshold of 1650 . Statistical significance is symbolized with * for $p<0.05(n=8-12)$

increased osteoprogenitor cell activity. The origin and lineage of these cells will be the topic of future investigation.

Next, to determine whether Jag1 can also increase bone regeneration in the appendicular skeleton, bicortical bone defects where made in mouse femora and plugged with Gelfoam grafts bound with Jag1 (GF-Jag1) or control (GF-Vh) and examined at two timepoints. A day 10, Jag1 had induced a $71.7 \%$ increase in cortical bone relative to GF-Vh to $0.092 \pm 0.007 \mathrm{~mm}^{3}(p=0.024)$. 


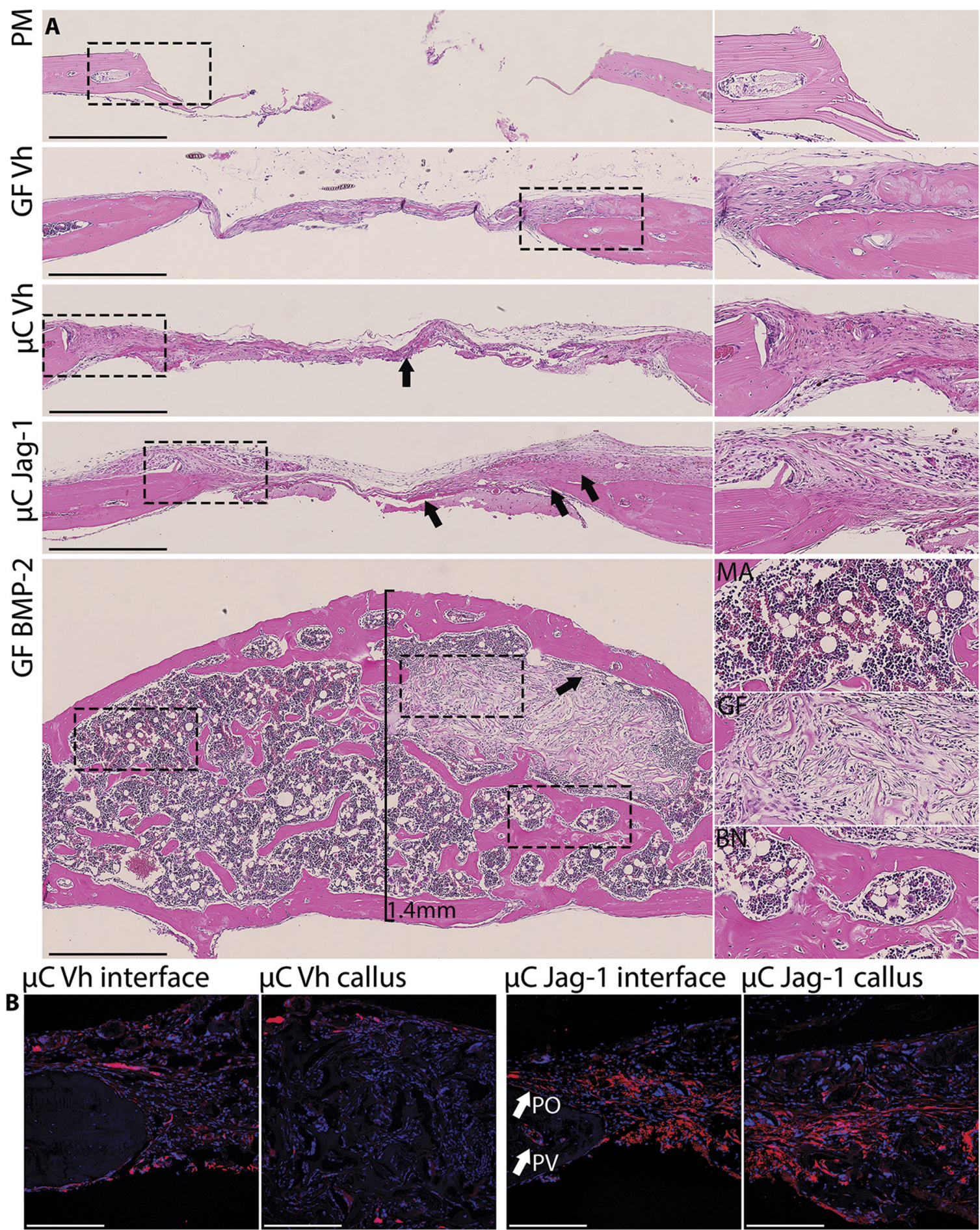

Fig. 4 Jag1 activates $\alpha$ SMA-positive mesenchyme in the calvaria without bone overgrowth. Representative H\&E histology (a) shows increased periosteal mesenchyme and improved bone matrix production 42 days post defect in the $\mu \mathrm{C}$-Jag 1 group (arrows) relative to the incomplete mineralization and fibrosis in the associated $\mu \mathrm{C}-\mathrm{Vh}$ control (arrow). BMP2 induced supraphysiological production of bone (BN) and fatty marrow (MA), encapsulating and preserving the Gelfoam scaffold (GF). Select ROls are zoomed adjacent to source images and marked using dashed lines. Scale bars are equal to $500 \mu \mathrm{m}$. $\alpha \mathrm{SMA}-\mathrm{Cre}^{\mathrm{ERT} 2}$; tdTomato reporter activity is increased in the $\mu \mathrm{C}$-Jag $1 \mathrm{group}$ relative to its $\mu \mathrm{C}$-Vh control (b). Confocal scale bars are equal to $200 \mu \mathrm{m}$

At day 20, this difference was increased to $91.1 \%, 0.290 \pm 0.062$ $\mathrm{mm}^{3}(p=0.009)$. There was a $40.0 \%$ increase in intramedullary bone at day 20 to $0.441 \pm 0.077 \mathrm{~mm}^{3}$ ( $p=0.042$ ) (Fig. 5). The GFJag1 group showed pronounced periosteal activation and increased new bone relative to contralateral GF-Vh controls.
Jag1 heals critical-sized calvarial defects in rats

Finally, we used a rat non-healing critical size defect to explore whether Jag1 could promote regeneration of non-healing injuries in a species other than mouse. $\mu \mathrm{CT}$ analysis of large non-healing calvarial defects in rats demonstrates the profound healing 

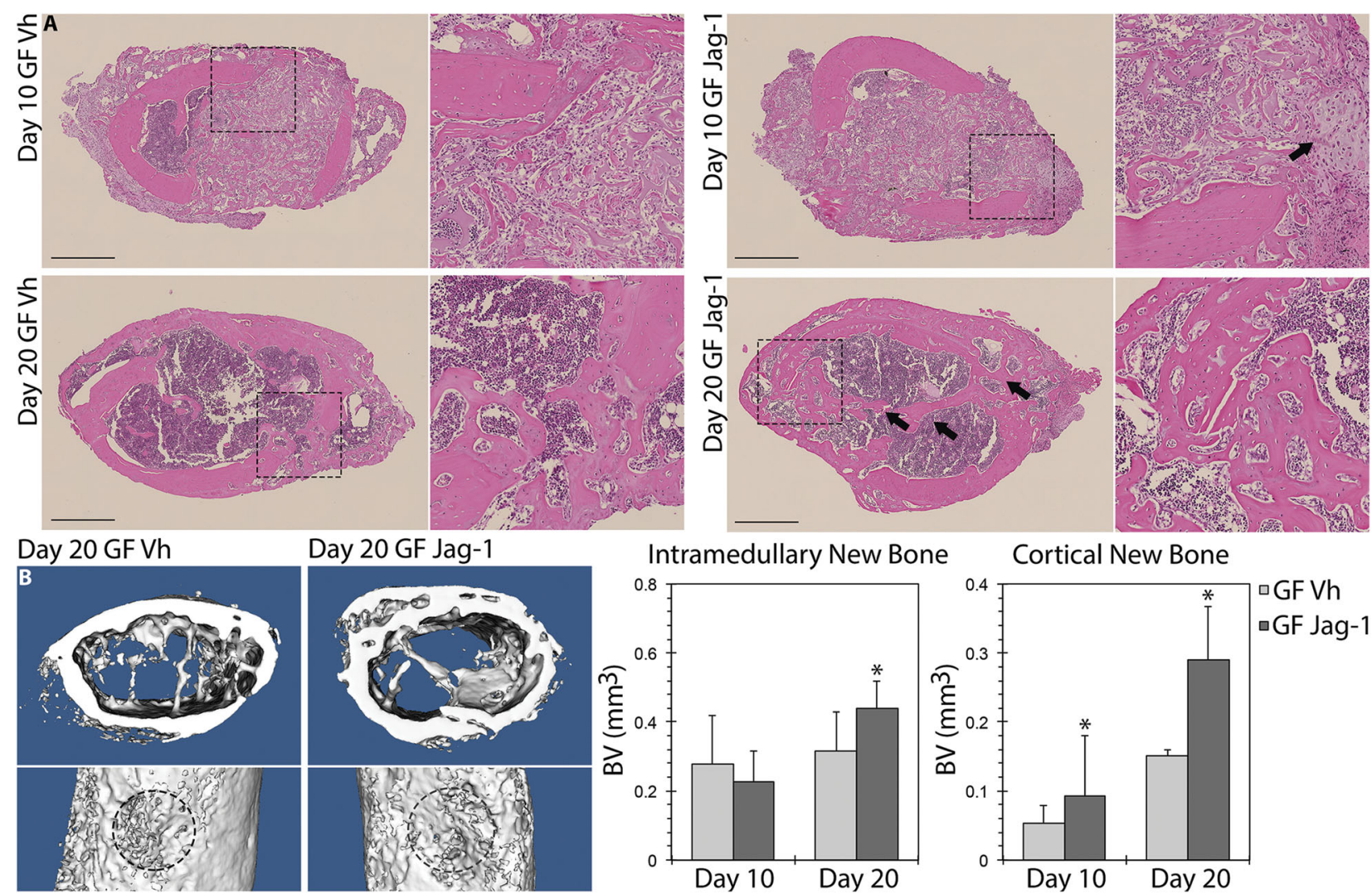

Fig. 5 Jag1 promotes healing of femoral defects. Representative H\&E histology (a) shows improved healing of Jag1-treated defects relative to contralateral GF-Vh controls. Selected ROls are zoomed adjacent to source images and marked using dashed lines. At day 10, local regions of endochondral ossification occur adjacent to residual Gelfoam and the cortical margin (arrow). The Jag1-induced increase in intramedullary and cortical bone is evident at day 20 (arrows). Scale bars are equal to $500 \mu \mathrm{m}$. $\mu \mathrm{CT}$ isosurfaces and data b were gathered at a threshold of 2667. Statistical significance is symbolized with ${ }^{*}$ for $p<0.05(n=5)$. Representative images represent matched sets from individual animals

potential of Jag1 (Fig. 6). Sixty days following surgery, total BV was $0.286 \pm 0.194 \mathrm{~mm}^{3}$ in the Vh group, $1.922 \pm 0.537 \mathrm{~mm}^{3}$ in the Jag 1 group $(671 \%$ of $V h, p<0.001)$ and $4.471 \pm 1.154 \mathrm{~mm}^{3}$ in the BMP2 group (1560\% of Vh, $p<0.001)$. Again, similar to observations with BMP2 in mice, BMP2 in rats resulted in hypertrophic bone extending above the margins of the surrounding bone, while Jag1 produced more regular bone that was within the plane of the original calvaria. Expression of the basic helix-loop-helix transcription factor HES1, a canonical Notch target gene, was increased $5.24 \pm 2.66$-fold at day $5(p=0.010)$, demonstrating that Jag 1 was functionally signaling.

\section{DISCUSSION}

There is an urgent need to develop new therapeutics for bone repair. While BMPs have proven to be potent tools and remain the clinical standard for promoting bone regeneration, over the past 5 years it has become clear that BMPs are not universally beneficial. Potential adverse effects of BMPs include inflammation, adipogenesis, ectopic/hypertrophic bone, radiculopathy and death. ${ }^{23}$ Additionally, BMPs can induce osteoclastogenesis in individuals with osteoporosis, exacerbating loss of mineral content and disqualifying a significant patient population. We demonstrate that the Notch signaling ligand Jag1 is required for maximal intramembranous bone regeneration and that delivery of Jag1 can promote new bone healing in both rats and mice.

In a marrow ablation model, which offers a method of studying intramembranous bone formation within a discrete physiological compartment on a condensed timeline, ${ }^{24}$ Notch signaling elements, particularly Jag1, were transcriptionally correlated with the differentiation of aSMA-positive mesenchyme to osteoblasts (COL2A1-negative, cartilage-free). In this model, cells transition from ACTA2-expressing progenitor cells (day 2) through RUNX2positive pre-osteoblasts (day 3) into IBSP-positive anabolic osteoblasts (days 3-4), which then rapidly fill the marrow cavity with bone. ${ }^{25}$ These mouse data corroborate a published microarray in rats, in which Jag1 was the most abundant ligand (upregulated 2.54-fold at day 5 post-ablation), Notch2 was the most abundant receptor and DLL4 showed the greatest increase in expression $\left(5.97-\right.$ fold). ${ }^{26}$ Perturbing Notch via tamoxifeninducible homozygous deletion of Jag1 in that mesenchymal lineage at the time of healing (aSMA-Cre ${ }^{\text {ERT2 }} ; J a g 1^{\mathrm{f} / \mathrm{f}}$ ), but not Jag2 $\left(\right.$ aSMA-Cre ${ }^{\text {ERT2 }} ; \mathrm{Jag}^{\mathrm{f} / \mathrm{f}}$ ), resulted in reduced bone regeneration.

Having identified a positive regulatory role of Jag1 in adult bone healing, we sought to therapeutically deliver Jag1 protein to the site of a calvarial defect in mice, which heals by intramembranous ossification. Notch (DLL4) gain-of-function has been proposed as a therapeutic paradigm for muscle healing, ${ }^{27}$ but recombinant Notch ligand has, until now, never been used to drive regeneration. Agarose Protein $G$ beads were previously used to deliver Jag1 in vitro, ${ }^{22}$ so a similar approach was adopted using $5 \mu \mathrm{m}$-diameter biodegradable PLGA microcarrier beads (rigid substrate for Jag1) embedded in Gelfoam (osteoconductive scaffold). This approach resulted in significantly greater defect coverage and BV versus microcarrier-laden vehicle by $\mu \mathrm{CT}$ 42-days post-defect. The positive control, BMP2, delivered at an approximately equimolar concentration, produced a complex boney mass, with retained Gelfoam and adipocyte-laden marrow, 
$\mathrm{Vh}$

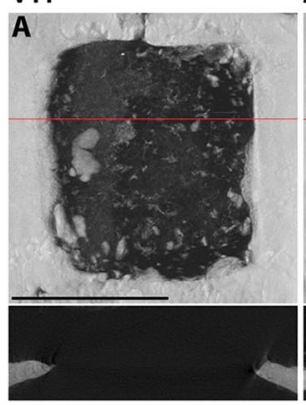

$\operatorname{Jag} 1$

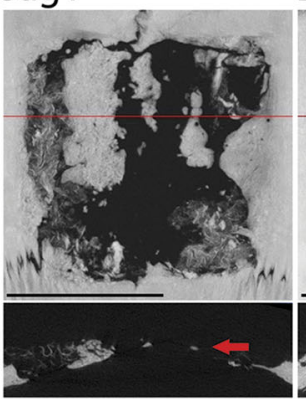

BMP2

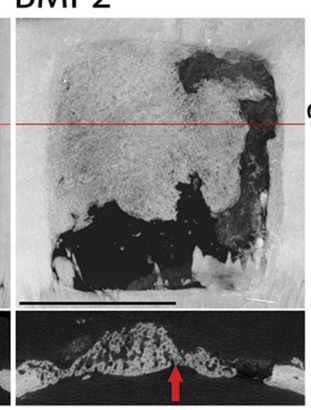

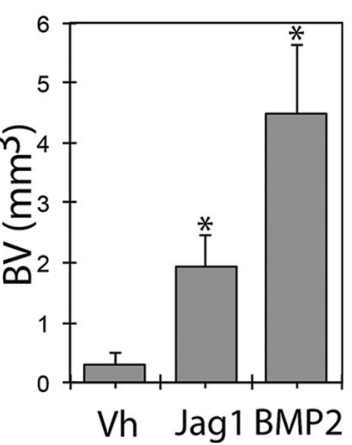

B

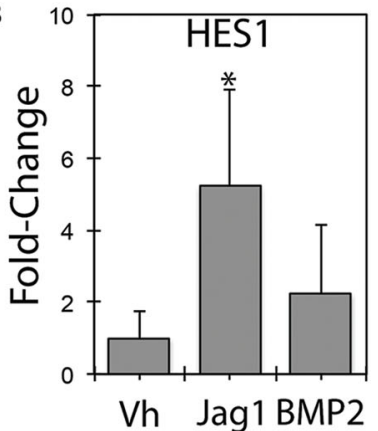

Fig. 6 Jag1 regenerates critical-sized calvarial defects in rats. Jag1 drives pronounced bone healing in rat segmental skull defects, as seen via $\mu C T$ (a). Representative dorsal 3D reconstructions are shown with 2D transverse sections (red lines) below. Jag1-induced healing occurs within the plane of surrounding bone (arrow), while BMP2 causes bone overgrowth above the injury margin: termed hypertrophy (arrow). Expression of the canonical Notch target gene, HES1, is increased in a frontal quadrant of rat calveriae relative to GAPDH in the Jag1-treated animals preceding this bone formation in the rostral portion of the healing defect $(\mathbf{b})$. Associated quantitation represents bone within $6.6 \times 6.6 \mathrm{~mm}$ ROIs centered in each defect. Scale bars are equal to $5 \mathrm{~mm}$. Statistical significance is symbolized with * for $p<0.05(n=4-6)$

protruding well above the surrounding parietal bones. The $\mu \mathrm{C}$ Jag1 group had $59.9 \%$ of the regenerated BV of the GF-BMP2 group, but importantly, the new bone remained in the plane of the defect, and was qualitatively more similar to the native cranial bone.

Overproduction of bone by BMPs, including potentially fatal ectopic bone and malignant transformation, ${ }^{23}$ is a clinical problem that we hope to overcome with targeted delivery of Notch ligands. While delivered in equimolar doses here, the high potency of BMP2 relative to Jag1 suggests that fine-tuning dose will be an important aspect of promoting a physiologically appropriate bone anabolic response. BMPs are osteoinductive in solution and migrate away from the application site. Unlike BMP2, Jag1 appears to promote a bone-anabolic phenotype only among cell types that are predisposed to form bone, such as post-injury mesenchyme: enhancing the safety profile. We hypothesize that Jag1 treatment will be broadly therapeutic, though ALGS patients, who are predisposed to pathologic femur fractures that heal poorly, likely stand to benefit more. Furthermore, collagen matrices including Gelfoam are also available in injectable form, increasing applicability of Jag1-functionalized scaffolds beyond those injuries requiring open reduction. Long-term safety studies of Notchactivating therapies in bone will be required to corroborate these hypotheses.

To demonstrate applicability of Jag1 therapy to the appendicular skeleton using a simpler clinical delivery methodology, Jag1 was adhered directly to Gelfoam (microcarrier-free) and then administered to the site of a bicortical window defect in the femur. This model is intended to be intramembranous, but does include some regions of endochondral ossification near the periosteal margins of the defects. Jag1 enhanced cortical and intramedullary ossification in this context. The most profound healing was observed in the largest, most clinically relevant injury, when Jag1 was delivered to critical, non-healing calvarial defects on Helistat sponges in rats. Mirroring the mouse data, the Jag1 group resulted in $43.0 \%$ of the regenerated BV of the BMP2 group, $671 \%$ more than vehicle control, but the new bone was formed inline with the native calvarial bone, without the hypertrophic phenotype. HES1 was upregulated in the Jag1 group, confirming the bioactivity of the recombinant protein via canonical Notch signaling in this model.

In future experiments it will be important to define the mechanistic basis of this improved healing. While results from our laboratory and others show that Jag1 and canonical Notch signaling can direct osteoblast differentiation in a homotypic manner, ${ }^{13}$ it is feasible that the effect of Jag1 extends beyond the mesenchymal lineage. Notch signaling regulates elements of vascular architecture that are essential for angiogenesis and the osteoprogenitor niche. ${ }^{28}$ Ectopic Jag1 can cause a primary vascular phenotype, in addition to altered mesenchymalendothelial crosstalk, ${ }^{29}$ which were not investigated in this study. Furthermore, with autografts functioning as the current standard of care for bone replacement, it will be important to characterize the osteoinductive efficacy of Jag1 on structural, load-bearing materials: ${ }^{30}$ particularly due to the mechanosensitivity of ligand presentation on receptor activation. ${ }^{31}$ Finally, fracture healing is significant translational goal of our approach-it will be important to determine whether or not Jag1-mediated Notch signaling has the same osteoblastic phenotype during chondrocyte transdifferentiation during endochondral ossification. ${ }^{32}$

In conclusion, homotypic Jag1-mediated canonical Notch signaling within adult mesenchymal progenitor cells promotes osteoblast differentiation and bone healing. This phenomenon is conserved across the craniofacial and appendicular skeleton and from mice to rats, and the therapy is effective with two different collagen scaffolds with or without the presence of microcarrier beads. Jag1 may be functioning to increase proliferation of earlystage progenitor cells, ${ }^{33}$ to promote late-stage differentiation and anabolic activity of osteoblasts, ${ }^{22}$ or both (Supplementary Fig. S5). Direct delivery of recombinant Jag1 to bone injuries represents a therapeutic approach for skeletal regeneration.

\section{MATERIALS AND METHODS}

Study design and animal use

All research was conducted following all relevant procedures and guidelines, under the guidance and approval of the Institutional Animal Care and Use Committees of the University of Michigan, Michigan State University or the University of Pennsylvania. Wild-type C57BL/6 mice and Sprague Dawley rats were enrolled in the reported studies. The following transgenic mouse strains on a C57BL/6 background were also used: aSMA$\mathrm{Cre}^{\text {ERT2 }}$,tdTomato, $^{25}$ Jag 1 $^{\mathrm{f} / \mathrm{f} / 34}$ aSMA-Cre ${ }^{\text {ERT2 }}$; Jag 1 ${ }^{\mathrm{f} / \mathrm{f}}$, Jag2 $2^{\mathrm{f} / \mathrm{f}, 35}$ aSMA-Cre ${ }^{\text {ERT2 }}$; $\mathrm{Jag}^{\mathrm{f} / \mathrm{f}}$. Recombination was induced by a single intraperitoneal injection of $75 \mathrm{mg} / \mathrm{kg}$ tamoxifen at time of surgery. Animals were socially housed when possible and allowed ad libitum access to food and water. Isoflurane gas (Abbott Labs) was used for inducing and maintaining surgical anesthesia under external heat and animals received subcutaneous $1 \mathrm{mg} / \mathrm{kg}$ Buprenorphine SR (ZooPharm) for post-operative analgesia. Incision sites were cleared of hair and disinfected with alternating scrubs of alcohol and povidone-iodine. Euthanasia was performed in accordance with current American Veterinary Medical Association guidelines. Surgeries and quantitative analyses were conducted with investigators blinded from timepoint, genotype and treatment. 


\section{Mouse femoral marrow ablation surgery}

Stifles were clipped and scrubbed and longitudinal incisions at the patella were created using sterile surgical instruments, exposing the intercondylar area of the femurs. 25-gauge needles were manually drilled through the intermedullary canal toward the proximal growth plate and repeatedly reamed. 26-gauge needles were then used to flush the cavity with saline until the discharge was clear. Incisions were closed with surgical glue. For gene expression analysis, 6-12 animals were used per time point, with each time point representing a 1:1 ratio of males to females. Six animals per timepoint ( $n=3$ males, $n=3$ females) were used for $\mu \mathrm{CT}$ analysis and histology. Four animals were used for fluorescence double-labeling and reporter activity. Mice in this experiment were $19 \pm 5$ weeks old and weighed $26 \pm 4 \mathrm{~g}$. For knockout data and associated controls, $n=3$ females mice were used per group, at $10.0 \pm 1.1$ weeks old and $20.7 \pm 1.1 \mathrm{~g}$.

\section{Mouse calvarial defect and grafting}

Absorbable gelatin sponges (Pfizer Gelfoam 12-7 mm, as above) were sliced longitudinally ( $1 \mathrm{~mm}$ thick) and cut into discs using a 4-mm biopsy punch (Integra 33-34). Collagen grafts were pre-hydrated in phosphatebuffered saline (PBS) overnight, expunged of fluid using a cotton tipped applicator (Puritan 806-WCL), placed into round bottom 96-well plates and hydrated with PBS (vehicle), $25 \mu \mathrm{g}$ of $5 \mu \mathrm{m}$-diameter green fluorescent PLGA microcarrier beads (Phosphorex Degradex) in PBS (microcarrier control), $10 \mu \mathrm{L}$ of $1.25 \mathrm{mg} / \mathrm{mL}$ recombinant human Jag1 (R\&D Systems 1277-JG) on $25 \mu \mathrm{g}$ microcarrier beads in PBS or $0.125 \mathrm{mg} / \mathrm{mL}$ recombinant human/mouse BMP-2 (R\&D Systems $355-B M$ ) in $800 \mathrm{nM} \mathrm{HCl}$ with $0.02 \%$ FBS in PBS two hours before the start of surgeries and incubated at $37^{\circ} \mathrm{C}$. Recipient mice in this study were $14 \pm 3$ weeks old and weighed $25 \pm 4 \mathrm{~g}$.

Parietal bones were exposed by single midline sagittal incisions and scraped of periosteum over the defect sites to facilitate drilling. Bilateral calvarial defects were then created using a Piezosurgery GP (Mectron) piezoelectric bone drill with an (3 mm-diameter) OT11 osteoplasty/ osteotomy insert with external saline irrigation, resulting in clean holes free of thermal damage with intact meninges. These critical size defects were immediately repaired using a graft from a single experimental group. All defects were produced by one author (D.W.Y.), all grafts were placed by another author (R.S.), and both of these individuals were blinded from the assigned treatment at time of surgery. Incisions were closed with surgical glue and animals were closely monitored during recovery.

\section{Mouse femoral defect and grafting}

US FDA-approved absorbable gelatin sponges (Pfizer Gelfoam 12-7 mm), $2 \mathrm{~mm}$ thick and cut using a $1.5 \mathrm{~mm}$ biopsy punch, were incubated for $45 \mathrm{~min}$ prior to the start of surgeries in $15 \mu \mathrm{L}$ of either PBS (vehicle) or $400 \mu \mathrm{g} / \mathrm{mL}$ recombinant rat Jag1 (R\&D Systems AF599) in PBS. The surgical sites were accessed by craniolateral incision and blunt dissection between the Rectus femoris and Vastus lateralis muscles. Femurs were elevated using forceps and through-and-through window defects were created in the distal metaphysis $5 \mathrm{~mm}$ proximal to the femoral trochleae using a Dremel 8100 with a $0.75 \mathrm{~mm}$ drill bit. Defects were plugged with collagen grafts from each group (left side vehicle, right side Jag1) and incisions were sutured shut. Ten animals received bilateral femoral window defects, with $n=5$ analyzed at day 10 and $n=5$ analyzed at day 20 .

\section{Rat calvarial defect and grafting}

Sprague Dawley rats received $7.0 \times 7.0 \mathrm{~mm}$ square critical-sized calvarial defects centered on the sagittal suture, using a piezoelectric bone drill with OP7 (periosteum) and OTS (osteotomy) inserts. $1.1 \times 1.1 \times 0.5$ $\mathrm{cm}$ absorbable collagen hemostatic sponge grafts (Helistat, Integra Life Sciences Corporation) were functionalized with PBS (vehicle), $50 \mu \mathrm{g}$ of recombinant human Jag1 or $5 \mu \mathrm{g}$ of recombinant human/mouse BMP-2 and placed within the defects. Incisions were closed with 4-0 nylon suture. Fourteen animals were harvested at 60 days and 12 animals were harvested at 5 days. Rats weighed $450-550 \mathrm{~g}$ and were 5-6 months of age.

\section{Sample preparation and histological analysis}

Tissues were harvested and fixed in $10 \%$ buffered formalin under agitation for $72 \mathrm{~h}$. Samples destined for fluorescence microscopy were then transferred to $30 \%$ sucrose in PBS under agitation for $48 \mathrm{~h}$ and then stored at $4{ }^{\circ} \mathrm{C}$. Samples destined for $\mu \mathrm{CT}$ and histology were transferred to
$70 \%$ ethanol and stored at $4{ }^{\circ} \mathrm{C}$. After $\mu \mathrm{CT}$ imaging, these bones underwent routine processing and embedding in paraffin, with hematoxylin and eosin slides prepared by the histology cores of Michigan State University or the University of Michigan Dental School. Slides were imaged on a Nikon Eclipse Ni upright microscope with DS-Ri2 camera and Prior OptiScan II motorized stage, scanned and stitched at 20x in MIS Elements BR software. Basic post-processing and figure design were conducted in Adobe Photoshop CS6.

\section{Micro-computed tomography $(\mu \mathrm{CT})$}

Ablated mouse femurs and mouse calveriae were scanned in GE Healthcare explore Locus instruments. Marrow-ablated femurs and calveriae were scanned in water at $18 \mu \mathrm{m}$ voxel size using the following settings: $80 \mathrm{kV}, 80 \mu \mathrm{A}, 1600 \mathrm{~ms}, 400$ views. Reconstruction and analysis was performed in MicroView (Parallax Innovations). The intramedullary $\mathrm{ROI}$ for each ablated femur was manually defined, consisting of $25 \%$ of the total femur length, shifted $5 \%$ proximal to the distal growth plate, at a threshold of 1650 . For mouse calvarial samples, BV contained within $3.33 \times 3.33 \times 1.66 \mathrm{~mm}$ ROls centered over each defect were quantified. Representative images were created using smoothened isosurfaces at these settings. For calvarial grafts, new BV was calculated by subtracting the BV contained within the ROI following creation of a fresh defect postmortem (PM), such that no healing had occurred. A low threshold of 666 was used here for comparing treatments with different-density bone. Comparisons between the $\mu \mathrm{C}-\mathrm{Vh}$ and $\mu \mathrm{C}$-Jag1 groups were further elaborated at the standard, more conservative threshold of 1650 using total quantitation within the ROI without subtraction of PM so as to allow for quantitation of density.

Mouse tibial defects were scanned in a Scanco $\mu \mathrm{CT} 35$ at $21 \mu \mathrm{m}$ voxel size using the following settings: $55 \mathrm{kV}$ energy, $145 \mu \mathrm{A}$ intensity, $650 \mathrm{~ms}$, 500 views. User-defined contours with automated interpolation were used to define ROls encompassing cortical bone, the defect and associated callus. Similarly, user-defined contours were drawn around the original cortical bone for exclusion. This semi-automated segmentation method analyzes the callus outside the pre-existing cortical bone. The callus was analyzed in Scanco software using Gaussian filtering with a global mineral threshold of $260 \mathrm{mgHA} / \mathrm{cm}^{3}$.

For rat calveriae, analysis was performed using a Bruker SkyScan 1076. Specimens were immersed in water and scanned individually at $9 \mu \mathrm{m}$ voxel size using the following settings: $65 \mathrm{KV}, 381 \mu \mathrm{A}, 200 \mathrm{~ms}, 600$ views. Images were reconstructed using NRecon software with attenuation coefficient 0.005534, smoothing 3.0, misalignment compensation 2.0, ring artifact reduction 10 , beam-hardening correction $30 \%$. DataViewer software was used to re-align the images and quantitative parameters were assessed using CTan software. Bone voxels were thresholded between $63-255$ by averaging auto-thresholds across each file. 3D analysis was done on $6.6 \times$ $6.6 \mathrm{~mm}$ area that was adjusted in the $z$-axis to encompass all new bone.

\section{Gene expression analysis}

For marrow ablation, intramedullary tissue was harvested by clipping the proximal and distal epiphyses and repeatedly reaming/flushing the diaphysis from both ends with $1 \mathrm{~mL}$ of TRlzol per animal using a $22 \mathrm{G}$ needle and syringe. For mouse calvarial defects, a 4-mm biopsy punch was used to collect tissue centered over each defect, which was subsequently flash-frozen in liquid nitrogen in TRlzol and homogenized in a cryogenic bead mill (Bertin Precellys). For rat calvarial defects, a frontal quadrant of each day 5 sample was processed for gene expression analysis. RNA was isolated by acid guanidinium thiocyanate-phenol-chloroform extraction with GlycoBlue coprecipitant followed by purification with Qiagen RNeasy Midi spin columns with on-column DNase digestion per the manufacturer's protocol, then reverse-transcribed. qPCR reactions were conducted with 20ng of template using custom primers (Table S1) and SYBR Select master mix for 40 cycles and analyzed using the $2^{\text {-ddCt }}$ method. Fold-change data is reported as mean \pm standard deviation relative to basal tissue.

\section{Fluorescence microscopy}

Conventional fluorescence images of $7 \mu \mathrm{m}$-thick fixed, non-decalcified, cryosectioned femurs from aSMA-Cre ${ }^{\mathrm{ERT} 2}$; tdTomato marrow-ablated mice were acquired on a Nikon Eclipse 50i POL microscope. Half of the animals received tamoxifen at time of surgery; all animals received subcutaneous injections of $10 \mathrm{mg} / \mathrm{kg}$ calcein (Sigma C0875) at time of surgery and $50 \mathrm{mg} /$ $\mathrm{kg}$ demeclocycline hydrochloride (Sigma D61400) at day three. Animals 
were sacrificed at day 4 and examined for label uptake and tdTomato fluorescence.

Confocal images of $9 \mu \mathrm{m}$-thick fixed, non-decalcified, coronal cryosections of grafted calveriae were acquired on a Nikon A1 + upright confocal microscope using a CFI Plan Apochromat $\lambda$ VC 20x air objective. Samples were collected from aSMA-Cre ${ }^{\text {ERT2 }}$;tdTomato mice and slides were costained with DAPI. A $403 \mathrm{~nm}$ laser was used to excite DAPI and a $560 \mathrm{~nm}$ laser was used to excite tdTomato. A 405/488/561/647 main dichroic mirror was used. Emission dichroic mirrors were used to separate emission wavelengths for each detector channel. Emission from DAPI was separated and filtered using a 505LP dichroic mirror and a 450/50BP filter. Emission from tdTomato was separated and filtered using a 560LP dichroic mirror and a 595/50BP filter. A 5 slice, $7 \mu \mathrm{m}$, z-series confocal image was acquired and a maximum intensity projection was created, with a $1024 \times 1024$ frame size and $2 \times$ Kalman averaging to decrease noise.

\section{Statistical analysis}

Data values are expressed as mean \pm standard deviation. Statistical significance was determined by two-way Student's $t$-tests versus basal, wild-type or vehicle controls. For qPCR data within the marrow ablation timecourse, suspected outliers ( $5 \%$ of $\mathrm{dCt}$ values) were screened via a twoway Dixon's Q-test or a Rosner's extreme Studentized deviate test with $p<$ 0.05 . No outliers were removed from other data sets. Points of significance are annotated graphically in figures with ${ }^{*} \equiv p<0.05$.

\section{Data availability}

The data sets generated during and/or analyzed during the current study are available from the corresponding author on reasonable request.

\section{ACKNOWLEDGEMENTS}

aSMA-Cre ${ }^{\text {ERT2 }}$ mice were kindly provided by Dr. Brya Matthews and Dr. Ivo Kalajzic. Dr. Clifford M. Les and Dr. Yadav Wagley provided valued discussion. Michele M. Lowe, Kaela L. Caballero, Katelyn E. Gagne, Hannah E. Sexton, Nick Servadio, and Cathy Tyler provided technical support. Funding was provided in part by the Department of Defense CDMRP W81XWH-15-1-0689 to K.D.H. K.D.H. and K.M.L. were partially supported by the Institute for Translational Medicine and Therapeutics (ITMAT) Transdisciplinary Program in Translational Medicine and Therapeutics at the University of Pennsylvania, NIH UL1RR024134. DWY is supported by $\mathrm{NIH}$ F32DE026346. RLZ is supported by NIH F30AR071201.

\section{AUTHOR CONTRIBUTIONS}

Experimental design: D.W.Y., R.S., M.H.M., K.D.H.; data acquisition: D.W.Y., R.S., R.L.X., J. D.B., A.L.L., D.R.Y., T.L.M., M.E.M., M.H.M., W.J.T.; analysis/interpretation: all authors; manuscript drafting: D.W.Y., K.D.H.; final approval: all authors.

\section{ADDITIONAL INFORMATION}

Supplementary information accompanies the paper on the npj Regenerative Medicine website (https://doi.org/10.1038/s41536-017-0037-9).

Competing interests: K.D.H. is the co-founder of Skelegen, LLC and holds US patent application 14/760,371. Other authors declare no competing financial interests.

Publisher's note: Springer Nature remains neutral with regard to jurisdictional claims in published maps and institutional affiliations.

\section{REFERENCES}

1. van Staa, T. P., Dennison, E. M., Leufkens, H. G. \& Cooper, C. Epidemiology of fractures in England and Wales. Bone 29, 517-522 (2001).

2. Antonova, E., Le, T. K., Burge, R. \& Mershon, J. Tibia shaft fractures: costly burden of nonunions. BMC Musculoskelet. Disord. 14, 42 (2013).

3. Mankin, H. J., Gebhardt, M. C., Jennings, L. C., Springfield, D. S. \& Tomford, W. W. Long-term results of allograft replacement in the management of bone tumors. Clin. Orthop. Relat. Res. 324, 86-97 (1996).

4. Burge, R. et al. Incidence and economic burden of osteoporosis-related fractures in the United States, 2005-2025. J. Bone Miner. Res. 22, 465-475 (2007).

5. Nikkel, L. E. et al. Impact of comorbidities on hospitalization costs following hip fracture. J. Bone Jt. Surg. 94, 9-17 (2012).
6. Carragee, E. J., Ghanayem, A. J., Weiner, B. K., Rothman, D. J. \& Bono, C. M. A challenge to integrity in spine publications: years of living dangerously with the promotion of bone growth factors. Spine J. 11, 463-468 (2011).

7. Artavanis-Tsakonas, S., Rand, M. D. \& Lake, R. J. Notch signaling: cell fate control and signal integration in development. Science 284, 770-776 (1999).

8. Oda, T. et al. Mutations in the human Jagged1 gene are responsible for Alagille syndrome. Nat. Genet. 16, 235-242 (1997).

9. Majewski, J. et al. Mutations in NOTCH2 in families with Hajdu-Cheney syndrome. Hum. Mutat. 32, 1114-1117 (2011).

10. Xue, Y. et al. Embryonic lethality and vascular defects in mice lacking the Notch ligand Jagged1. Hum. Mol. Genet. 8, 723-730 (1999).

11. Dong, Y. et al. RBPjk-dependent Notch signaling regulates mesenchymal progenitor cell proliferation and differentiation during skeletal development. Development 137, 1461-1471 (2010).

12. Hilton, M. J. et al. Notch signaling maintains bone marrow mesenchymal progenitors by suppressing osteoblast differentiation. Nat. Med. 14, 306-314 (2008).

13. Youngstrom, D. W. et al. Jagged1 expression by osteoblast-lineage cells regulates trabecular bone mass and periosteal expansion in mice. Bone 91, 64-74 (2016).

14. Yorgan, T. et al. Osteoblast-specific Notch2 inactivation causes increased trabecular bone mass at specific sites of the appendicular skeleton. Bone 87, 136-146 (2016).

15. Engin, F. et al. Dimorphic effects of Notch signaling in bone homeostasis. Nat. Med. 14, 299-305 (2008).

16. Dishowitz, M. I., Terkhorn, S. P., Bostic, S. A. \& Hankenson, K. D. Notch signaling components are upregulated during both endochondral and intramembranous bone regeneration. J. Orthop. Res. 30, 296-303 (2012).

17. Dishowitz, M. I. et al. Systemic inhibition of canonical Notch signaling results in sustained callus inflammation and alters multiple phases of fracture healing. PLoS. One. 8, e68726 (2013).

18. Matthews, B. G. et al. Analysis of aSMA-labeled progenitor cell commitment identifies notch signaling as an important pathway in fracture healing. J. Bone Miner. Res. 29, 1283-1294 (2014).

19. Wang, C. C. et al. NOTCH signaling in skeletal progenitors is critical for fracture repair. J. Clin. Investig. 126, 1471-1481 (2016).

20. Small, D. et al. Soluble Jagged 1 represses the function of its transmembrane form to induce the formation of the Src-dependent chord-like phenotype. J. Biol. Chem. 276, 32022-32030 (2001).

21. Dishowitz, M. I. et al. Jagged1 immobilization to an osteoconductive polymer activates the Notch signaling pathway and induces osteogenesis. J. Biomed. Mater. Res. A 102, 1558-1567 (2014).

22. Zhu, F., Sweetwyne, M. T. \& Hankenson, K. D. PKC $\delta$ Is required for Jagged-1 induction of human mesenchymal stem cell osteogenic differentiation. Stem Cells 31, 1181-1192 (2013).

23. James, A. W. et al. A review of the clinical side effects of bone morphogenetic protein-2. Tissue Eng. Part. B Rev. 22, 284-297 (2016).

24. Bragdon, B., Lybrand, K. \& Gerstenfeld, L. Overview of biological mechanisms and applications of three murine models of bone repair: closed fracture with intramedullary fixation, distraction osteogenesis, and marrow ablation by reaming. Curr. Protoc. Mouse Biol. 5, 21-34 (2015).

25. Grcevic, D. et al. In vivo fate mapping identifies mesenchymal progenitor cells. Stem Cells 30, 187-196 (2012).

26. Wise, J. K. et al. Temporal gene expression profiling during rat femoral marrow ablation-induced intramembranous bone regeneration. PLoS One 5, e12987 (2010).

27. Conboy, I. M., Conboy, M. J., Smythe, G. M. \& Rando, T. A. Notch-mediated restoration of regenerative potential to aged muscle. Science 302, 1575-1577 (2003).

28. Kusumbe, A. P., Ramasamy, S. K. \& Adams, R. H. Coupling of angiogenesis and osteogenesis by a specific vessel subtype in bone. Nature 507, 323-328 (2014).

29. Gonzalez-King, H. et al. Hypoxia inducible factor-1alpha potentiates Jagged 1mediated angiogenesis by mesenchymal stem cell-derived exosomes. Stem Cells 35, 1747-1759 (2017).

30. McDermott, A. M., Mason, D. E., Lin, A. S., Guldberg, R. E. \& Boerckel, J. D. Influence of structural load-bearing scaffolds on mechanical load-and BMP-2-mediated bone regeneration. J. Mech. Behav. Biomed. Mater. 62, 169-181 (2016).

31. Meloty-Kapella, L., Shergill, B., Kuon, J., Botvinick, E. \& Weinmaster, G. Notch ligand endocytosis generates mechanical pulling force dependent on dynamin, epsins, and actin. Dev. Cell 22, 1299-1312 (2012).

32. Hu, D. P. et al. Cartilage to bone transformation during fracture healing is coordinated by the invading vasculature and induction of the core pluripotency genes. Development 144, 221-234 (2017).

33. Lawal, R. A. et al. The Notch ligand Jagged1 regulates the osteoblastic lineage by maintaining the osteoprogenitor pool. J. Bone Miner. Res. 32, 1320-1331 (2017).

34. Loomes, K. M. et al. Bile duct proliferation in liver-specific Jag1 conditional knockout mice: effects of gene dosage. Hepatology 45, 323-330 (2007). 
35. Xu, J., Krebs, L. T. \& Gridley, T. Generation of mice with a conditional null allele of the Jagged2 gene. Genesis 48, 390-393 (2010).

cc (i) Open Access This article is licensed under a Creative Commons Attribution 4.0 International License, which permits use, sharing, adaptation, distribution and reproduction in any medium or format, as long as you give appropriate credit to the original author(s) and the source, provide a link to the Creative Commons license, and indicate if changes were made. The images or other third party material in this article are included in the article's Creative Commons license, unless indicated otherwise in a credit line to the material. If material is not included in the article's Creative Commons license and your intended use is not permitted by statutory regulation or exceeds the permitted use, you will need to obtain permission directly from the copyright holder. To view a copy of this license, visit http://creativecommons. org/licenses/by/4.0/.

(c) The Author(s) 2017 